\title{
Effectiveness evaluation of polarization in OAM multiplexing
}

\author{
Yuki Ito $^{1}$, Shuhei Saito ${ }^{1}$, Hirofumi Suganuma ${ }^{1}$, Kayo Ogawa ${ }^{2}$, \\ and Fumiaki Maehara ${ }^{1, \text { a) }}$ \\ ${ }^{1}$ Graduate School of Fundamental Science and Engineering, \\ Waseda University, 3-4-1 Ohkubo, Shinjuku-ku, Tokyo 169-8555, Japan \\ ${ }^{2}$ Graduate School of Science, Japan Women's University, \\ 2-8-1 Mejirodai, Bunkyo-ku, Tokyo 112-8681, Japan
}

a)fumiaki_m@waseda.jp

Abstract: This paper investigates the system capacity of polarized orbital angular momentum (OAM) multiplexing by comparing it with OAM multiplexing without polarization for the same number of antenna elements. Since the same number of antenna elements is assumed for both approaches, their performances can be compared under the same number of simultaneous OAM streams, thereby allowing the effectiveness of polarization on UCA-based OAM multiplexing to be examined. Numerical results, obtained through computer simulations, provide the mode selectivity and system capacity with respect to link distance and carrier frequency.

Keywords: orbital angular momentum (OAM), uniform circular array (UCA), mode multiplexing, polarization, system capacity

Classification: Wireless Communication Technologies

\section{References}

[1] M. Shafi, A.F. Molisch, P.J. Smith, T. Haustein, P. Zhu, P.D. Silva, F. Tufvesson, A. Benjebbour, and G. Wunder, "5G: A tutorial overview of standards, trials, challenges, deployment, and practice," IEEE J. Sel. Areas Commun., vol. 35, no. 6, pp. 1201-1221, June 2017. DOI: 10.1109/jsac.2017.2692307

[2] Z. Zhang, Y. Xiao, Z. Ma, M. Xiao, Z. Ding, X. Lei, G.K. Karagiannidis, and P. Fan, "6G wireless networks: Vision, requirements, architecture, and key technologies," IEEE Veh. Technol. Mag., vol. 14, no. 3, pp. 28-41, Sept. 2019. DOI: $10.1109 /$ mvt.2019.2921208

[3] Z. Pi, J. Choi, and R. Heath, "Millimeter-wave gigabit broadband evolution toward 5G: Fixed access and backhaul," IEEE Commun. Mag., vol. 54, no. 4, pp. 138-144, April 2016. DOI: 10.1109/mcom.2016.7452278

[4] D. Lee, H. Sasaki, H. Fukumoto, K. Hiraga, and T. Nakagawa, "Orbital angular momentum (OAM) multiplexing: An enabler of a new era of wireless communications," IEICE Trans. Commun., vol. E100-B, no. 7, pp. 1044-1063, July 2017. DOI: $10.1587 /$ transcom.2016sci0001

[5] A. Trichili, K.-H. Park, M. Zghal, B.S. Ooi, and M.-S. Alouini, "Communicating using spatial mode multiplexing: Potentials, challenges, and perspectives," IEEE Commun. Surveys Tut., vol. 21, no. 4, pp. 3175-3203, 2019. DOI: 10.1109/comst.2019.2915981 
[6] S.M. Mohammadi, L.K.S. Daldorff, J.E.S. Bergman, R.L. Karlsson, B. Thidé, K. Forozesh, T.D. Carozzi, and B. Isham, "Orbital angular momentum in radio: A system study," IEEE Trans. Antennas Propag., vol. 58, no. 2, pp. 565-572, Feb. 2010. DOI: 10.1109/tap.2009.2037701

[7] L. Sung, D. Park, and D.-H. Cho, "Performance analysis of orbital angular momentum (OAM) transmission using dual polarization antenna based uniform circular array architecture," Proc. 2016 13th IEEE Annu. Consum. Commun. Netw. Conf. (CCNC 2016), pp. 517-522, Jan. 2016. DOI: 10.1109/ccnc. 2016.7444834

[8] G.-T. Gil, J.Y. Lee, D.-H. Cho, S. Jung, and J. Kang, "Dual polarized UCAbased OAM multi-mode transmission with inter-mode spreading," Proc. 2018 IEEE 87th Veh. Technol. Conf. (VTC-Spring 2018), pp. 1-5, June 2018. DOI: 10.1109/vtcspring.2018.8417588

[9] S. Saito, Y. Ito, H. Suganuma, K. Ogawa, and F. Maehara, "Performance analysis of polarized OAM multiplexing considering the effect of polarization interference," Proc. 2020 IEEE Int. Conf. Commun. Workshops (ICC Workshops 2020), pp. 1-6, June 2020. DOI: 10.1109/iccworkshops49005.2020.9145214

[10] M. Hirabe, Z. Ryuji, H. Miyamoto, K. Ikuta, and E. Sasaki, "40 m transmission of OAM mode and polarization multiplexing in E-band," Proc. 2019 IEEE Globecom Workshops (GC Wkshps 2019), pp. 1-6, Dec. 2019. DOI: 10.1109/gcwkshps45667.2019.9024495

[11] H. Sasaki, Y. Yagi, T. Yamada, T. Semoto, and D. Lee, "An experimental demonstration of over $100 \mathrm{Gbit} / \mathrm{s}$ OAM multiplexing transmission at a distance of $100 \mathrm{~m}$ on $40 \mathrm{GHz}$ band," Proc. 2020 IEEE Int. Conf. Commun. Workshops (ICC Workshops 2020), pp. 1-6, June 2020. DOI: 10.1109/ iccworkshops49005.2020.9145429

\section{Introduction}

In recent years, the demand for mobile broadband services has increased exponentially [1]. Fifth-generation (5G) mobile systems are designed to provide a thousandfold capacity increase over $4 \mathrm{G}$ for future services, including the internet of things (IoT) and intelligent transport systems (ITS) [1]. Moreover, in the sixth-generation (6G) mobile communications systems, which are aimed to be realized by 2030, a peak data rate of $1 \mathrm{~Tb} / \mathrm{s}$, which is 100 times that of the $5 \mathrm{G}$ network, is expected to be provided for a large-dimensional and autonomous network that integrates space, air, ground, and underwater networks [2]. To achieve such advanced mobile systems that support gigabit or terabit connections, it is necessary to upgrade small cell backhaul as well as enhance access link technologies [3].

For small cell backhaul, line-of-sight (LoS) wireless communication with fixed transmitter and receiver locations is more attractive than fiber solutions in terms of flexibility, scalability, and capital expenditure [3]. In this context, orbital angular momentum (OAM) multiplexing, where the phase front twists along the propagation direction, is becoming of increasing interest owing to its potential to simultaneously transmit multiple data streams through a single aperture pair $[4,5]$.

In the generation of OAM signals, a uniform circular array (UCA) enables simultaneous transmission of multiple modes and can be regarded as a practical approach for realizing OAM multiplexing [4, 6]. Moreover, OAM signals can 
adopt polarization multiplexing as well as mode multiplexing to enhance the system capacity further $[7,8,9,10,11]$. Several meaningful studies have analytically $[7,8,9]$ and experimentally $[10,11]$ investigated polarized OAM multiplexing. Especially in $[7,8]$, the effectiveness of polarization on UCA-based OAM multiplexing has been analytically investigated in comparison with traditional OAM multiplexing at a constant transmission power. However, in these previous studies, the total number of antenna elements was assumed to be double that of the traditional scheme; thus, the impact of polarization itself on the system capacity could not be understood and remains unclear.

Considering the background described above, we investigate the system capacity of polarized OAM multiplexing in comparison with traditional OAM multiplexing without polarization for the same number of antenna elements. In our study, as both approaches have the same number of antenna elements, a direct performance comparison under a constant number of OAM streams and a constant transmit power can be conducted. Numerical results, obtained through computer simulation, provide the mode selectivity and system capacity with respect to link distance and carrier frequency, thereby clarifying the effectiveness of the polarization itself.

\section{Effect of polarization on OAM multiplexing}

Fig. 1 shows the comparison, in terms of system configuration, of the traditional UCA-based OAM multiplexing and that with polarization. In Fig. 1, the total number of antenna elements is set to $2 N$; thus, the total number of OAM streams is $2 N$, regardless of the use of polarization. In application of polarization, the number of antenna elements allocated in each polarization plane is halved compared with that in the traditional OAM multiplexing, which reduces the number of multiplexed OAM modes $L$ to $N$ and decreases the received power. However, lower modes, which have strong robustness against beam divergence [4], can be utilized on both the vertical and the horizontal planes.

As shown in Fig. 1, the transmit signal vector with polarization $\mathbf{s} \in \mathbb{C}^{2 N}$ is
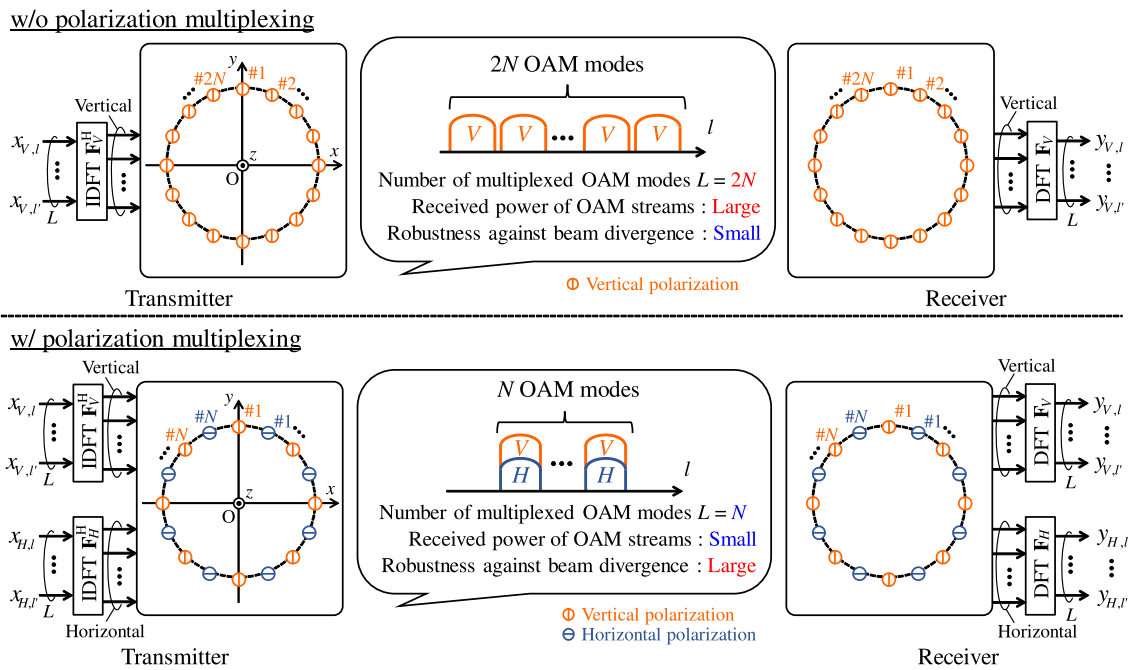

Fig. 1. Comparison of the traditional UCA-based OAM multiplexing and that with polarization. 
expressed as

$$
\begin{aligned}
\mathbf{s} & =\mathbf{F}^{\mathrm{H}} \mathbf{x}, \\
\mathbf{F}^{\mathrm{H}} & =\operatorname{diag}\left\{\mathbf{F}_{V}^{\mathrm{H}}, \mathbf{F}_{H}^{\mathrm{H}}\right\},
\end{aligned}
$$

where $\mathbf{x} \in \mathbb{C}^{2 L}$ is the modulated signal vector. Moreover, $\mathbf{F}_{V}^{\mathrm{H}}$ and $\mathbf{F}_{H}^{\mathrm{H}} \in \mathbb{C}^{N \times L}$ are the inverse discrete Fourier transform (IDFT) matrices of the transmit UCA considering the direction of polarization, where " $V$ " and " $H$ " represent the vertical and horizontal polarization, respectively.

At the receiver, the received signal vector $\mathbf{y} \in \mathbb{C}^{2 L}$ is represented by

$$
\begin{aligned}
\mathbf{y} & =\mathbf{F}(\mathbf{H s}+\mathbf{n})=\mathbf{F H F}^{\mathrm{H}} \mathbf{x}+\tilde{\mathbf{n}}, \\
\mathbf{H} & =\left[\begin{array}{ll}
\mathbf{H}_{V, V} & \mathbf{H}_{V, H} \\
\mathbf{H}_{H, V} & \mathbf{H}_{H, H}
\end{array}\right], \\
\mathbf{F} & =\operatorname{diag}\left\{\mathbf{F}_{V}, \mathbf{F}_{H}\right\},
\end{aligned}
$$

where $\mathbf{H}_{V, V}, \mathbf{H}_{V, H}, \mathbf{H}_{H, V}, \mathbf{H}_{H, H} \in \mathbb{C}^{N \times N}$ and $\mathbf{F}_{V}, \mathbf{F}_{H} \in \mathbb{C}^{L \times N}$ are the channel matrices and the discrete Fourier transform (DFT) matrices of the received UCA considering the direction of the polarization, respectively. Moreover, $\mathbf{n} \in \mathbb{C}^{2 N}$ and $\tilde{\mathbf{n}} \in \mathbb{C}^{2 L}$ are the noise vectors, and their elements follow the same distribution. Assuming free-space propagation, the channel condition between the $m$-th transmit antenna element and the $n$-th received antenna element in the UCA is expressed by [7]

$$
h_{n, m}=\frac{\lambda}{4 \pi d_{n, m}} e^{-j 2 \pi d_{n, m} / \lambda} \cdot \mathbf{P}\left(\theta_{n, m}^{\prime}, \phi_{n, m}^{\prime}\right)^{\mathrm{T}} \cdot \mathbf{P}\left(\theta_{n, m}, \phi_{n, m}\right),
$$

where $\lambda$ is wavelength and $d_{n, m}$ is distance between the transmit and received antenna elements, respectively. Moreover, $\theta_{n, m}, \theta_{n, m}^{\prime}$ and $\phi_{n, m}, \phi_{n, m}^{\prime}$ are the elevation and the azimuth angles, and $\mathbf{P}\left(\theta_{n, m}, \phi_{n, m}\right)$ and $\mathbf{P}\left(\theta_{n, m}^{\prime}, \phi_{n, m}^{\prime}\right)$ are the radiation pattern vectors of the transmit and received antenna elements, respectively. Assuming the isotropic antenna as an antenna element, the radiation pattern vectors of these antennas are given by [7]

$$
\mathbf{P}_{i}(\theta, \phi)=\left\{\begin{array}{ll}
{[1,0]^{\mathrm{T}}} & (V \text {-polarization }) \\
{[0,1]^{\mathrm{T}}} & (H \text {-polarization })
\end{array} .\right.
$$

It should be noted that the radiation pattern in Eq. (7) indicates that the orthogonality among different polarization planes is perfectly retained.

From Eq. (3), the received signals of the $l$-th OAM mode with vertical and horizontal polarization $y_{V, l}, y_{H, l}$ are given by

$$
\begin{aligned}
& y_{V, l}=\sigma_{V, l} x_{V, l}+\tilde{n}_{V, l}, \\
& y_{H, l}=\sigma_{H, l} x_{H, l}+\tilde{n}_{H, l},
\end{aligned}
$$

where $x_{V, l}, x_{H, l}$ and $\tilde{n}_{V, l}, \tilde{n}_{H, l}$ are the modulated signal and noise, respectively. Moreover, $\sigma_{V, l}$ and $\sigma_{H, l}$ are $(l, l)$ elements of $\mathbf{F}_{V} \mathbf{H}_{V, V} \mathbf{F}_{V}^{\mathrm{H}}$ and $\mathbf{F}_{H} \mathbf{H}_{H, H} \mathbf{F}_{H}^{\mathrm{H}}$, respectively. 
From Eqs. (8) and (9), the signal-to-noise ratios (SNRs) of the $l$-th OAM mode with vertical and horizontal polarization $\gamma_{V, l}, \gamma_{H, l}$ are represented by

$$
\begin{gathered}
\gamma_{V, l}=\frac{E\left[\left|\sigma_{V, l} x_{V, l}\right|^{2}\right]}{E\left[\left|\tilde{n}_{V, l}\right|^{2}\right]}=\frac{\left|\sigma_{V, l}\right|^{2} P_{V, l}}{P_{n}}, \\
\gamma_{H, l}=\frac{E\left[\left|\sigma_{H, l} x_{H, l}\right|^{2}\right]}{E\left[\left|\tilde{n}_{H, l}\right|^{2}\right]}=\frac{\left|\sigma_{H, l}\right|^{2} P_{H, l}}{P_{n}},
\end{gathered}
$$

where $P_{V, l}$ and $P_{H, l}$ are the transmit power and $P_{n}$ is the noise power, respectively.

Assuming the radiation pattern in Eq. (7), polarized OAM multiplexing involves the summation of the system capacities of the vertical and horizontal planes, as given by

$$
C_{\text {sum }}=\sum_{l \in\{-N / 2+1, \cdots, N / 2\}}\left(\log _{2}\left(1+\gamma_{V, l}\right)+\log _{2}\left(1+\gamma_{H, l}\right)\right) .
$$

On the other hand, the system capacity of the traditional OAM multiplexing without polarization can be calculated in the same manner as that for the polarized case described above. In the traditional case, all the antenna elements are assigned to either vertical or horizontal polarization. Therefore, the system capacity of the traditional UCA-based OAM can be calculated as

$$
C_{\text {sum }}=\left\{\begin{array}{cc}
\sum_{l \in\{-N+1, \cdots, N\}} \log _{2}\left(1+\gamma_{V, l}\right) & (V \text {-polarization }) \\
\sum_{l \in\{-N+1, \cdots, N\}} \log _{2}\left(1+\gamma_{H, l}\right) & (H \text {-polarization })
\end{array} .\right.
$$

\section{Numerical results}

In this section, we evaluate the effectiveness of polarization on UCA-based OAM multiplexing in terms of the SNR and system capacity using the variable of link distance. In our performance evaluation, the total number of antenna elements in a UCA is set to 64, and the isotropic antenna is taken as an antenna element. The radius of UCA, total transmit power and noise power are set to $R=0.30 \mathrm{~m}, P_{t}=23 \mathrm{dBm}$ and $P_{n}=-80 \mathrm{dBm}$, respectively. In the case of polarization multiplexing, the number of multiplexed OAM modes is set to $L=32$ in both vertical and horizontal planes, respectively. On the other hand, in the traditional approach without polarization, the number of multiplexed OAM modes is set to $L=64$ in only vertical plane. Moreover, the transmission power is the same among all the OAM streams.

Fig. 2 shows the SNR of each OAM mode, where the carrier frequency $f_{c}=$ $28 \mathrm{GHz}$, and link distance $z=1 \mathrm{~m}$ and $10 \mathrm{~m}$. From Fig. 2, it can be observed that higher modes in the traditional approach retain a relatively high SNR, as the effect of beam divergence is relatively small in such a short link distance of $z=1 \mathrm{~m}$. By contrast, in the case of $z=10 \mathrm{~m}$, the SNRs in higher modes are significantly degraded, regardless of the use of polarization multiplexing, because the effect of beam divergence becomes large for relatively long link distance. Moreover, the SNRs of polarization multiplexing become $6 \mathrm{~dB}$ lower than those in the traditional approach. This is because the number of transmit and received antenna elements allocated in each polarization plane is halved compared with that in the traditional approach. 


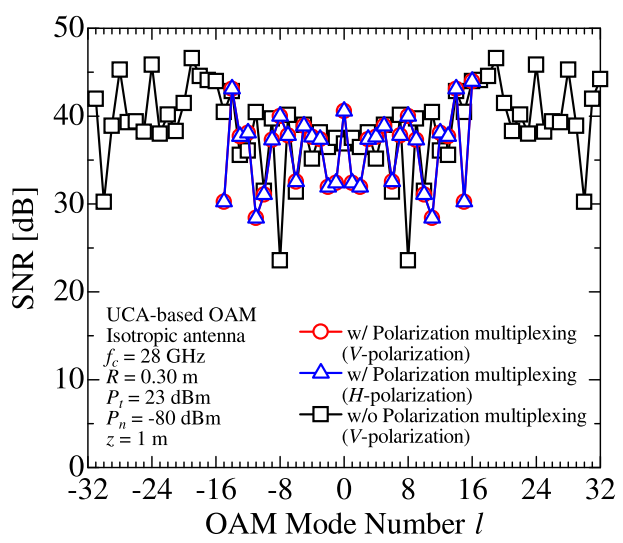

(a) $z=1 \mathrm{~m}$

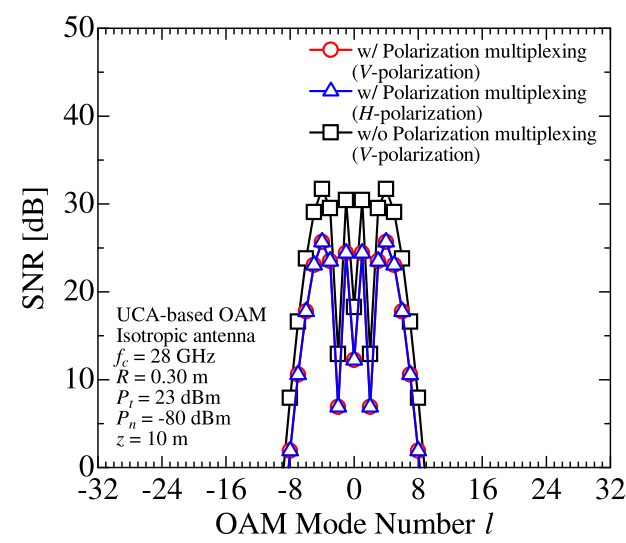

(b) $z=10 \mathrm{~m}$

Fig. 2. SNR of each OAM mode.

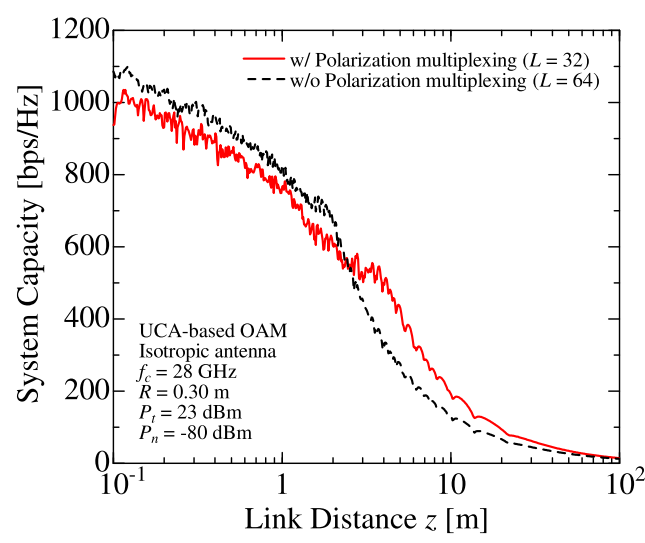

(a) $f_{c}=28 \mathrm{GHz}$

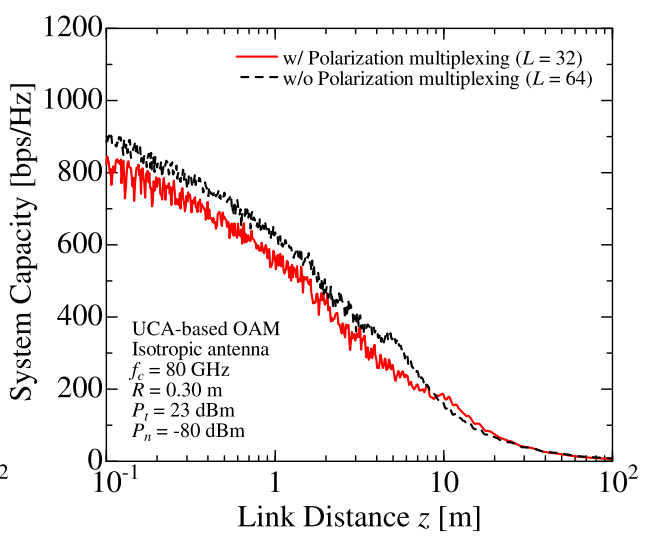

(b) $f_{c}=80 \mathrm{GHz}$

Fig. 3. System capacity versus link distance.

Fig. 3 shows the system capacity versus link distance $z$, where the carrier frequency $f_{c}=28 \mathrm{GHz}$ and $80 \mathrm{GHz}$. From Fig. 3, it can be observed that, for a relatively short link distance, the traditional approach without polarization provides better system capacity than that with polarization. This is because higher modes are available for relatively short link distance, as presented in Fig. 2. By contrast, for a relatively long link distance, the system capacity of polarization multiplexing surpasses that of the traditional approach because the available modes are restricted owing to the large beam divergence, and both vertical and horizontal planes can effectively utilize the lower modes. Furthermore, with an increase in the carrier frequency $f_{c}$, the traditional approach enlarges the region of $z$ surpassing the case of polarization multiplexing owing to less beam divergence.

\section{Conclusion}

In this paper, we investigated the system capacity of polarized OAM multiplexing by comparing it with traditional OAM multiplexing without polarization for the same number of antenna elements and the same transmission power. Since the same number of antenna elements was used in both cases, their performances could be directly compared under the same number of simultaneous OAM streams. The 
numerical results indicated that the traditional approach provided better system capacity than the polarized case for a short link distance because of the large number of available modes. On the other hand, for a relatively long link distance, the increase in beam divergence makes polarized OAM multiplexing that can utilize lower modes on two different planes more effective. As for the effect of increasing carrier frequency, higher modes become available owing to less beam divergence, indicating that the traditional approach without polarization becomes more effective than that with polarization.

\section{Acknowledgments}

This work was supported by a Research Grant from the Telecommunications Advancement Foundation. 